\title{
SOME PROBLEMS ON NONLINEAR OSCILLATIONS
}

\author{
NGUYEN VAN DAO \\ Vietnam National University, Hanoi \\ 19 Le Thanh Tong, Hanoi, Vietnam \\ (This paper has been published in: \\ IUTAM Symposium on Recent Development in Non-linear Oscillations \\ of Mechanical Systems, Hanoi, 2000)
}

\section{INTRODUCTION}

Thia paper presents our research on the interaction between nolinear oscillations, Van-der-Pol's systems subjected to complicated excitations and quasilinear oscillations in systems with large static deflections [1-4]. It is well known that there is always an interaction of some kind between nonlinear oscillations, namely, between the forced, parametric and self-excited oscillators. Each of these oscillators demonstrates definite sustained oscillations, comprising one or a combination of several modes. The principal questions to be answered are: What will happen if these oscillators are coupled in some manner? Does a resultant nonlinear oscillation exist and is it stable? The satationary oscillations and their stability have been paid special attentions.

\section{INTERACTION BETWEEN EXTERNAL AND PARAMETRIC EXCITATIONS}

In this section, we examine some quasilinear oscillating systems subjected to external and parametric excitations. We restrict ourselves to a class of quasilinear systems with two excitations. The following systems have been considered:

The system with external excitations in principal resonance and parametric excitation of the first degree in subharmonic resonance of the order one-half:

$$
\ddot{x}+\omega^{2} x=\varepsilon\left\{\Delta x-h \dot{x}-\gamma x^{3}+2 p x \cos 2 \omega t+e \cos (\omega t+\sigma)\right\} .
$$

The system with interaction between an external excitation and a parametric excitation of the second degree, both in the principal resonance:

$$
\ddot{x}+\omega^{2} x=\varepsilon\left\{\Delta x-h \dot{x}-\gamma x^{3}+2 p x^{2} \cos \omega t+e \cos (\omega t+\gamma)\right\} .
$$

The system with interaction between an external excitation in principal resonance and a parametric excitation in subharmonic resonance:

$$
\ddot{x}+\omega^{2} x=\varepsilon\left\{\Delta x-h \dot{x}-\gamma x^{3}+2 p x^{3} \cos 2 \omega t+e \cos (\omega t+\sigma)\right\} .
$$

The interaction between two parametric excitations of the first and third degree:

$$
\ddot{x}+\omega^{2} x=\varepsilon\left\{\Delta x-h \dot{x}-\gamma x^{3}+2 p x \cos 2 \omega t+2 q x^{3} \cos 2(\omega t+\sigma)\right\} .
$$


The interaction between two parametric excitations of the first and second degree:

$$
\ddot{x}+\omega^{2} x=\varepsilon\left\{\Delta x-h \dot{x}-\gamma x^{3}+2 p x \cos 2 \omega t+2 q x^{2} \cos 2 \omega t\right\} .
$$

We now examine in more detail the equation (2.3) for $\sigma=0$. Its solution is found in the form [1]:

$$
x=a \cos \psi, \quad \dot{x}=-a \omega \sin \psi, \quad \psi=\omega t+\theta .
$$

The averaged equations are

$$
\left\{\begin{aligned}
\dot{a} & =d \frac{-\varepsilon}{2 \omega} f_{0}=\frac{-\varepsilon}{2 \omega}\left\{h \omega a+\frac{1}{2} p a^{3} \sin 2 \theta+e \sin \theta\right\}, \\
a \dot{\theta} & =\frac{-\varepsilon}{2 \omega} g_{0}=\frac{-\varepsilon}{2 \omega}\left\{\left(\Delta-\frac{3 \gamma}{4} a^{2}\right) a+p a^{3} \cos 2 \theta+e \cos \theta\right\} .
\end{aligned}\right.
$$

The amplitude and phase of stationary oscillations satisfy the equations:

$$
f=\varepsilon f_{0}-p a^{3} f_{1}=0, \quad g=e g_{0}+2 p a^{3} g_{1}=0 ;
$$

where

$$
\left\{\begin{array}{l}
f_{0}=h \omega a+\frac{1}{2} p a^{3} \sin 2 \theta+e \sin \theta=0 \\
g_{0}=\left(\Delta-\frac{3 \gamma}{4} a^{2}\right) a+p a^{3} \cos 2 \theta+e \cos \theta=0 \\
f_{1}=f_{0} \cos \theta-g_{0} \sin \theta, g_{1}=f_{0} \sin \theta+g_{0} \cos \theta
\end{array}\right.
$$

We can write

$$
\left\{\begin{array}{l}
f=A \sin \theta+B \cos \theta-E=0 \\
g=G \sin \theta+H \cos \theta-K=0
\end{array}\right.
$$

where

$$
\begin{aligned}
& A=e^{2}-p a^{4}\left[p a^{2}-\left(\Delta-\frac{3 \gamma}{4} a^{2}\right)\right]=T+p a^{4} X, \\
& X=\Delta-\frac{3 \gamma}{4} a^{2}+3 p a^{2}, \quad T=e^{2}-4 p^{2} a^{6}, \\
& H=e^{2}+2 p a^{4}\left[p a^{2}+\left(\Delta-\frac{3 \gamma}{4} a^{2}\right)\right]=T+2 p a^{4} X, \\
& B=-2 p h \omega a^{4}, \quad E=-e h \omega a, \quad G=4 p h \omega a^{4}, \quad K=-e a X .
\end{aligned}
$$

The transformation of the original equations $\left(f_{0}, g_{0}\right)$ into the associated ones $(f, g)$ hats the matrix:

$$
\{\tau\}=\left\{\begin{array}{cc}
e-2 p a^{3} \cos \theta & p a^{3} \sin \theta \\
4 p a^{3} \sin \theta & e+2 p a^{3} \cos \theta
\end{array}\right\} .
$$

The matrix has an important characteristic; its determinant denoted by $T$ depends only on $a$ (and aloso $\Delta$, in general)

$$
T=|\{\tau\}|=e^{2}-4 p^{2} a^{6} .
$$

Hence, in the plane $R\left(\Delta, a^{2}\right)$ we can identify two regions:

- The equivalence region satisfying $T \neq 0$ or $a^{6} \neq \frac{e^{2}}{4 p^{2}}$.

- The non-equivalence regions determined by $T=0$ or $a^{6}=\frac{e^{2}}{4 p^{2}}=a_{\gamma}^{6}$. 
In the equivalence region, the original equations $\left(f_{0}, g_{0}\right)$ and the associated equations $(f, g)$ are equivalent. Therefore, the corresponding parts of the original resonance curve $C_{0}$ and the associated resonance curve $C$ coincide.

The non-equivalence region is only a line. For the system under consideration, it is a straight line which is parallel to the abscissa axis $\Delta$ with the ordinate $a_{\gamma}^{2}$. The nonequivalnce line is a branch of the associated resonance curve $C$. It is not a branch of the original resonance curve. Almost all of this curve contains strange elements which belong to $C$, but do not belong to $C_{0}$. The method for determining the original resonance curve $C_{0}$ is to determine the associated resonance curve $C$ then exclude the strange elements. Resonance curves for the systems with and without friction are presented in Fig. 1 (for $h=0)$ and Fig. 2, 3 (for $h=0.003)$ and $(h=0.27)$. The amplitude curves in Fig. 1, 2 are similar to those of the interaction between linearly parametric and forced osscillation [1].

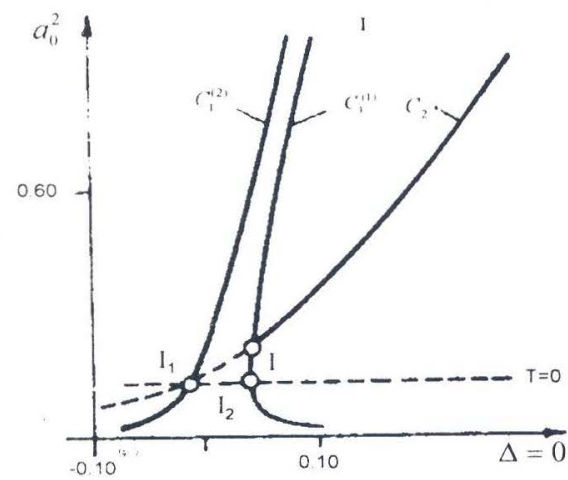

Fig. 1. Resonance curves for the system without friction

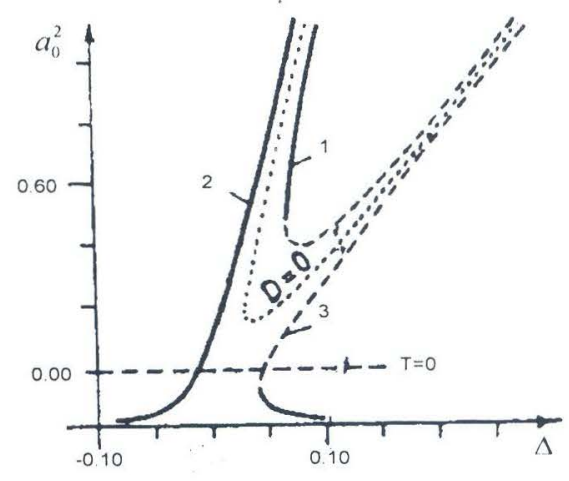

Fig. 2.. Resonance curves for the system with friction $h=0.01$

The amplitude curves in Fig. 3 characterize the nonlinear under consideration. For small values of amplitude $a_{0}$, the forced component dominated the other components and the corresponding parts of resonance curves are similar to those of forced oscillation. For large values of $a_{0}$ the influence of the parametric component is clear and as a result of the interaction between two oscillations, the resonance curve has the form of a upward parabola.

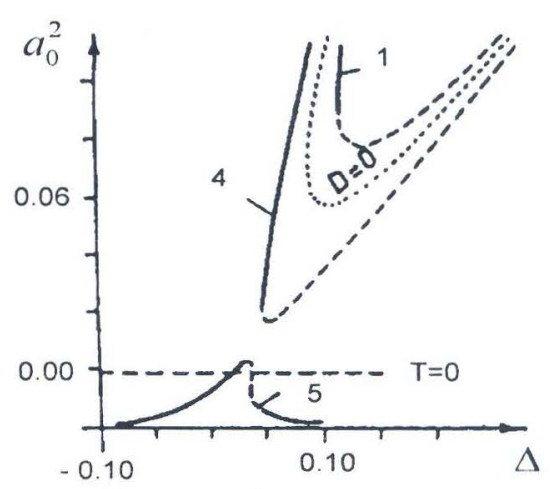

Fig. 3. Resonance curves for the system with friction $h=0.027$ 
Typical resonance curves for oscillating systems are given in Fig. 4 (for equation (2.1), Fig. 5 (for equation (2.2)), Fig. 6 (for equation (2.4)) and Fig. 7 (for equation (2.5)).

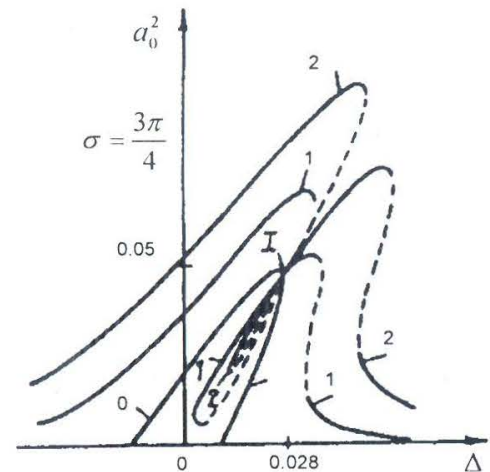

Fig. 4. Resonance curves for equation (2.1)

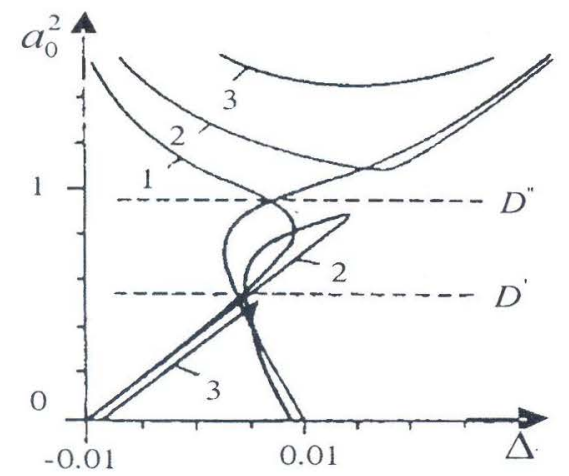

Fig. 6. Resonance curves for equation (2.4)

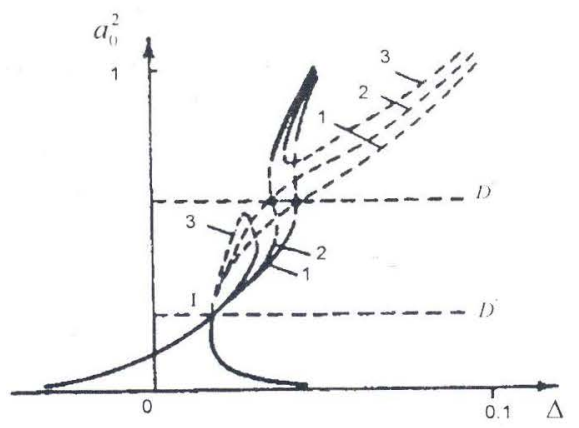

Fig. 5. Resonance curves for equation (2.2)

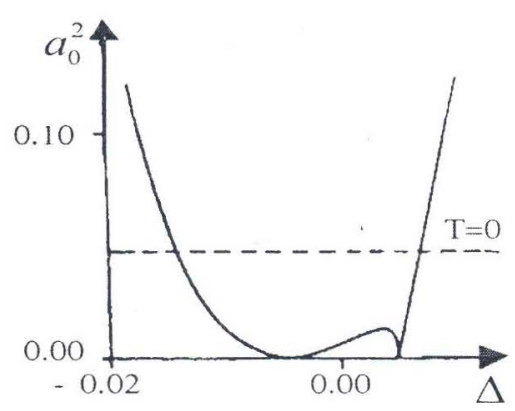

Fig. 7. Resonance curves for equation (2.5)

\section{VAN-DER-POL'S SYSTEMS SUBJECTED TO COMPLICATED EXCITATIONS}

Different kinds of resonance curves of stationary processes and the intermediate forms of the resonance curves have been examined with the aid of a computer. The system under consideration are:

Van-der-Pol's system under the parametric excitation of the first degree and forced external excitation described by d.e.:

$$
\ddot{x}+\omega^{2} x=\varepsilon\left\{\Delta x+h\left(1-k x^{2}\right) \dot{x}+2 p x \cos 2 \omega t+e \cos (\omega t+\sigma)\right\} .
$$

Typical forms of the resonance curves are shown in Figs. 8 and 9.

Van-der-Pol system subjected to the parametric excitation of the second degree and external excitation:

$$
\ddot{x}+\omega^{2} x=\varepsilon\left\{\Delta x+h\left(1-k x^{2}\right) \dot{x}+2 p x^{2} \cos \omega t+e \cos (\omega t+\sigma)\right\} .
$$




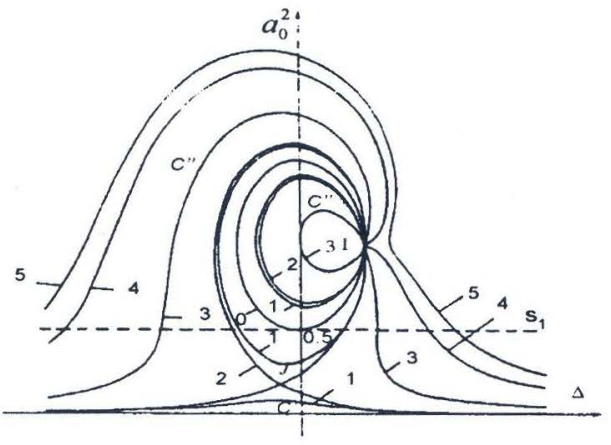

Fig. 8. Resonance curves for $\sigma=0$ and for $e=0$ (curve 0$), \quad e=0.0150$ (curve 1$)$, $e=0.0177$ (curve 2), $e=0.0500$ (curve 3 ), $e=0.1000$ (curve 4), $e=0.1200$ (curve 5).

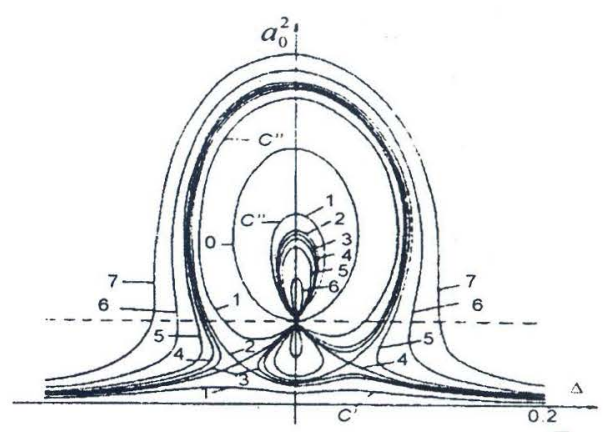

Fig. 9. Resonance curves for $\sigma=\frac{\pi}{4}$ and for $e=0$ (curve 0$), \quad e=0.0400$ (curve 1 ), $e=0.0483$ (curve 2), $e=0.0500$ (curve 3), $e=0.0516$ (curve 4), $e=0.0550$ (curve 5), $e=0.0648$ (curve 6), $e=0.0980$ (curve 7).

Typical resonance curves are given in Figs. 10 and 11.

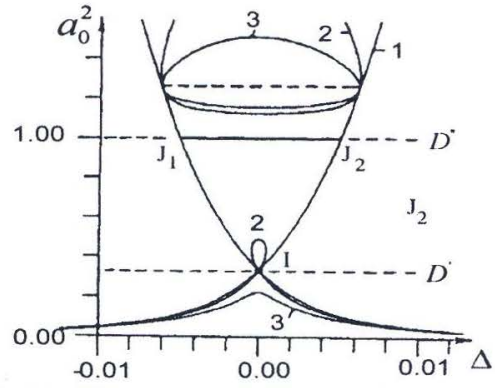

Fig. 10. Resonance curves for $\sigma=\pi$ and $h=0$ (curve 1), $h=0.03$ (curve 2), $h=$ 0.05 (curve 3 )

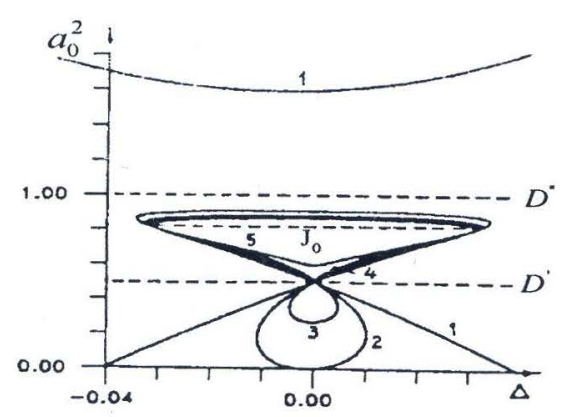

Fig. 12. Resonance curves for $p=0.05, q=$ $0.01,4<k<8$ and $h=0.300$ (curve 1), $h=0.0500$ (curve 2), $h=0.550$ (curve 3), $h=0.0666$ (curve 4$), h=0.800$ (curve 5)

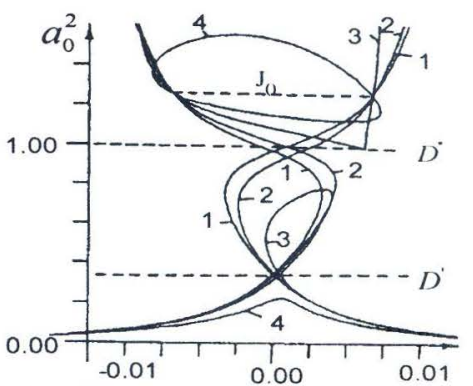

Fig. 11. Resonance curves for $\pi \neq \sigma$ $\in\left\{\frac{5 \pi}{6}, \frac{7 \pi}{6}\right\}$ and $h=0$ (curve 1 ), $h=$ 0.006 (curve 2), $h=0.02$ (curve 3 ), $h=$ 0.05 (curve 4)

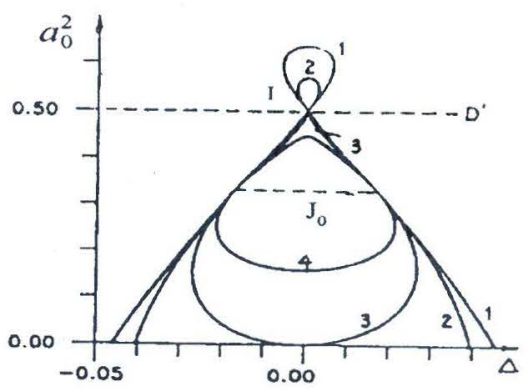

Fig. 13. Resonance curves for $k=8$ and $h=0.04$ (curve 1), $h=0.05$ (curve 2), $h=0.06$ (curve 3 ) 
Van-der-Pol's system subjected to the parametric excitations of the first and third degrees:

$$
\ddot{x}+\omega^{2} x=\varepsilon\left\{\Delta x+h\left(1-k x^{2}\right) \dot{x}+2 p x \cos 2 \omega t-2 q x^{3} \cos 2 \omega t\right\} .
$$

Some typical resonance curves are presented in Figs. 12 and 13.

Van-der-Pol's system with variable nonlinear friction described by d.e.:

$$
\ddot{x}+\omega^{2} x=\left\{\omega \Delta x+h\left[1-k(x+q \cos \omega t)^{2}\right] \dot{x}\right\} .
$$

Typical resonance curves are shown in Fig. 14

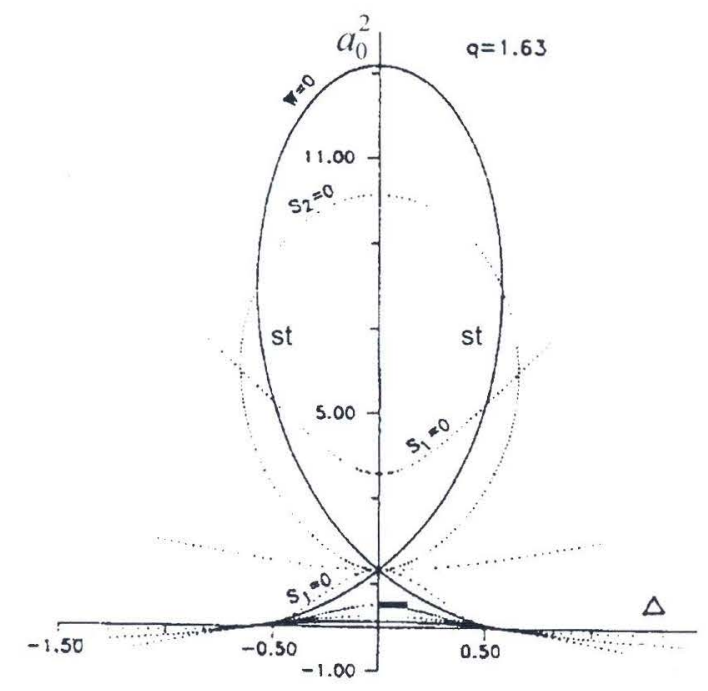

Fig. 14. Typical resonance curve of equation (3.4), where "st" is stable branch.

\section{QUASILINEAR OSCILLATIONS IN SYSTEMS WITH LARGE STATIC DEFLECTIONS}

In mechanical systems the static deflection of the elastic elements is usually not apparent in the equations of motion. The reason is that either a linear model of the elastic elements or an assumption of too small static deflection was accepted. In the present section both a nonlinear model of elastic elements and their large static deflection are considered, so that the nonlinear terms in the equation of motion appear with different degrees of smallness. In this case the nonlinearity of the system depends not only on the nonlinear characteristic of the elastic element but on its static deflection.

Let us consider the simplest oscillatory system which consists of a mass $M$ and a spring as shown in Fig. 15. The spring supporting the mass is assumed to be nonlinear with the characteristic

$$
f(u)=c_{0} u+\beta_{0} u^{3},
$$

so that the spring force acting on the mass $M$ is

$$
c_{0}(\Delta-x)+\beta_{0}(\Delta-x)^{3},
$$


where $\Delta$ is the deformation of the spring in the static equilibrium position. When $x=0$, the spring force $c_{0} \Delta+\beta_{0} \Delta^{3}$ is equal to the gravitational force $M g$. That is:

$$
c_{0} \Delta+\beta_{0} \Delta^{3}=M g .
$$

We have the equation of the mass $M$ in the form:

$$
M \ddot{x}+c_{0} x+3 \beta_{0} \Delta^{2} x-3 \beta_{0} \Delta x^{2}+\beta_{0} x^{3}=0 .
$$

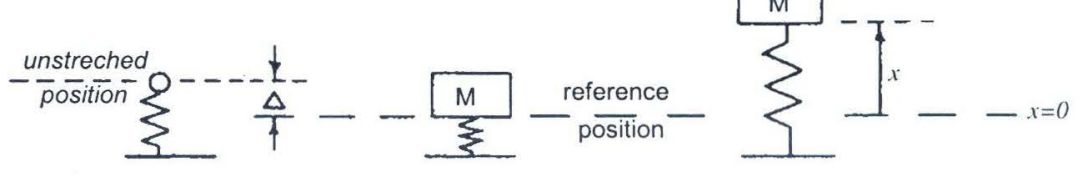

Fig. 15. Oscilatory system with large static deflection

It is supported that $\Delta$ is large and $x$ small enough, so that in comparision with the linear term, $\beta_{0} x^{3}$ is a small quantity of second degree and $\beta_{0} \Delta x^{2}$ is of the first degree of smallness:

$$
\frac{x}{\Delta}=0(\varepsilon), \quad \beta_{0} x^{3}=0\left(\varepsilon^{2}\right), \quad \beta_{0} \Delta x^{2}=0(\varepsilon),
$$

where $\varepsilon$ is a small positive parameter. In this case $\beta_{0} \Delta x^{2}$ is finite. Taking into account the viscous damping $h_{0} \dot{x}$ and the exciting force $P(t, x)$ which are both assumed to be small quantities of second degree and introducing the notation:

$$
\begin{gathered}
\omega^{2}=\frac{c_{0}+3 \beta_{0} \Delta^{2}}{M}, \quad \varepsilon \gamma=\frac{3 \beta_{0} \Delta}{M}, \quad \varepsilon^{2} \beta=\frac{\beta_{0}}{M}, \\
\varepsilon^{2} h=\frac{h_{0}}{M}, \quad \varepsilon^{2} f(t, x)=\frac{1}{M} P(t, x),
\end{gathered}
$$

we can write the equation of motion of the mass in the form:

$$
\ddot{x}+\omega^{2}=\varepsilon \gamma x^{2}-\varepsilon^{2}\left(h \dot{x}+\beta x^{3}-f(t, x)\right) .
$$

In comparision with the classical Duffing's equation, in equation (4.3) the small terms appear with different degrees; most of them are of second degree of smallness. From the structure of the equation (4.3) one can predict that the influence of the force on the motion of the mass $M$ can be found in the second approximation of the solution. A more general equation has also been investigated:

$$
\ddot{x}+\omega^{2} x=\varepsilon \gamma x^{2}+\varepsilon^{2} F(\tau, \varphi(\tau), x, \dot{x}) .
$$

The most interesting phenomenon in the systems under considerations is that their nonlinearity depends not only on the nonlinear characteristic of the spring as in the classical theory, but also $\mathrm{n}$ the static deflection $\Delta$. Namely, if the spring has soft characteristic $(\beta<0)$ (curve 3, Fig. 16), then the system under consideration also belongs to the soft type with more soft characteristic, because

$$
\alpha=\frac{3}{4} \beta-\frac{5 \gamma^{2}}{6 \omega^{2}}<0 .
$$


When the spring has hard characteristic $(\beta>0)$, the system under consideration belongs to the hard type if $\alpha>0$ or $c_{0}>7 \beta_{0} \Delta^{2}$ (curve 1, Fig. 16) and to the soft type if $c_{0}<7 \beta_{0} \Delta^{2}$ and to the neutral type if $c_{0}=7 \beta_{0} \delta^{2}$ (cureve 2, Fig. 16)

In addition to (4.4) the following problems have been considered:

The effect of $\varepsilon^{2}$-order due to the interaction between the excitation of $\varepsilon$-order in systems described by the equations:

$$
\begin{aligned}
& \ddot{x}+\omega^{2} x=\varepsilon\left\{h\left(1-k x^{2}\right) \dot{x}+e \cos 3 \omega t\right\}+\varepsilon^{2}\left(\Delta x-\gamma x^{3}\right), \\
& \ddot{x}+\omega^{2} x=\varepsilon[e \cos (2 \omega t+\chi)+2 p x \cos \omega t]+\varepsilon^{2}\left(\Delta x-2 h \dot{x}-\gamma x^{3}\right), \\
& \ddot{x}+\omega^{2} x=\varepsilon(e+2 p x \cos \omega t)+\varepsilon^{2}\left(\Delta x-h \dot{x}-\gamma x^{3}\right) .
\end{aligned}
$$

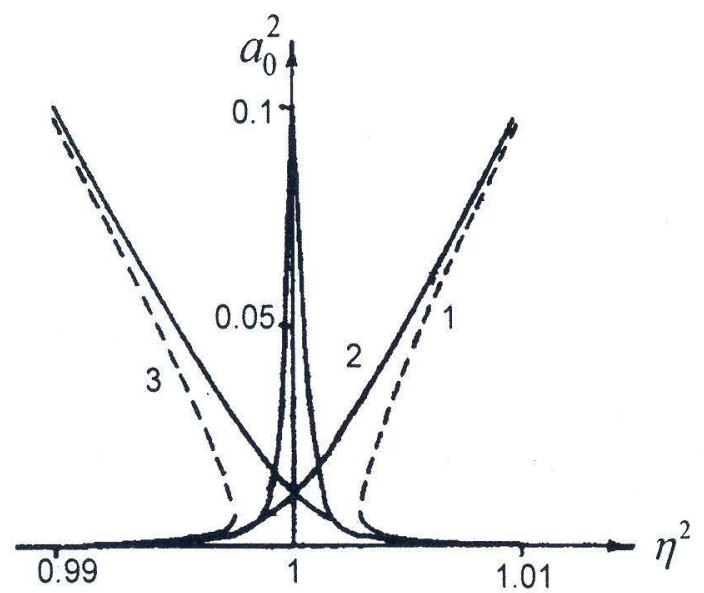

Fig. 16. Stationary resonance curves of equation (4.3).

The effect of $\varepsilon^{2}$-order caused by the interaction between the nonlinear restoring element and parametric excitation of $\varepsilon$-order in systems:

$$
\begin{aligned}
& \ddot{x}+\omega^{2} x=\varepsilon\left(\beta x^{2}+2 p x \cos \omega t\right)+\varepsilon^{2}\left(\Delta x-h \dot{x}-\gamma x^{3}\right), \\
& \ddot{x}+\omega^{2} x=\varepsilon\left(\beta x^{2}+e \cos 2 \omega t\right)+\varepsilon^{2}\left(\Delta x-h \dot{x}-\gamma x^{3}\right), \\
& \ddot{x}+\omega^{2} x=\varepsilon\left(\Delta x-\gamma x^{3}+e \cos 3 \omega t\right)+\varepsilon^{2}(-h \dot{x}) .
\end{aligned}
$$

The interaction of elements with two different orders in systems:

$$
\ddot{x}+\omega^{2} x=\varepsilon h\left(1-k x^{2}\right) \dot{x}+\varepsilon^{2}\left[\Delta x-\gamma x^{3}+f_{i}(t, x)\right],
$$

$f_{1}=2 p t \cos 2 \omega t, \quad f_{2}=e \cos \omega t ;$

$$
\begin{aligned}
\ddot{x}+\omega^{2} x & =2 \varepsilon p x \cos \omega t+\varepsilon^{2}\left[\Delta x-\gamma x^{3}+h\left(1-k x^{2}\right) \dot{x}\right], \\
\ddot{x}+\omega^{2} x & =\varepsilon\left(\alpha x^{2}+q \cos 2 \omega t\right)+\varepsilon^{2}\left[\Delta x+D\left(1-\delta x^{2}\right) \dot{x}-\beta x^{3}\right], \\
\ddot{x}+\omega^{2} x & =2 p \varepsilon x \cos \omega t+\varepsilon^{2}[\Delta x-h \dot{x}+2 q x \cos 2(\omega t+\sigma)], \\
\ddot{x}+\omega^{2} x & =\varepsilon\left(\alpha x^{2}+q \cos 2 \omega t\right)+\varepsilon^{2}\left[\Delta x-2 h \dot{x}-\beta x^{3}+r \cos (\omega t-\eta)\right], \\
\ddot{x}+\omega^{2} x & =\varepsilon p x \cos \omega t+\varepsilon^{2}\left[\Delta x-2 h \dot{x}-\beta x^{3}+r \cos (\omega t-\eta)\right], \\
\ddot{x}+x & =\varepsilon\left[\alpha x^{2}+q \cos 2 \varphi(t)\right]-\varepsilon^{2}\left(2 h \dot{x}+\beta x^{3}\right) .
\end{aligned}
$$




\section{CONCLUSION}

In this report, the interaction between external and parametric excitations, Van-derPol's systems subjected to complicated excitations and quasilinear oscillations in systems with large static deflections have been studied. The asymptotic method in combination with the numerical method and a computer have been used to study the stationary oscillations and their stability. The amplitude frequency curves (resonance curves) of the systems under consideration are various, and the nonlinear characteristics are markedelly changed in both quality and quantity in comparision with classical systems.

\section{REFERENCES}

1. Yu. A. Mitropolskii, Nguyen Van Dao, Applied Asymptotic Methods in Nonlinear Oscillations, Kluwer Academic Publishers, 1997.

2. Nguyen Van Dao, Nguyen Van Dinh, Tran Kim Chi, Interaction between nonlinear parametric and forced oscillations, Vietnam Journal of Mechanics, NCNST of Vietnam, T.XX, 1998, No 3.

3. Nguyen Van Dao, Quasilinear oscillations in systems with large static deflections, Proceedings of the International Conference on Applied Dynamics, Hanoi, 1995.

4. Nguyen Van Dao, Nguyen Van Dinh, Interaction between Nonlinear Oscillations, Vietnam National University Publishing House, Hanoi, 1999. 